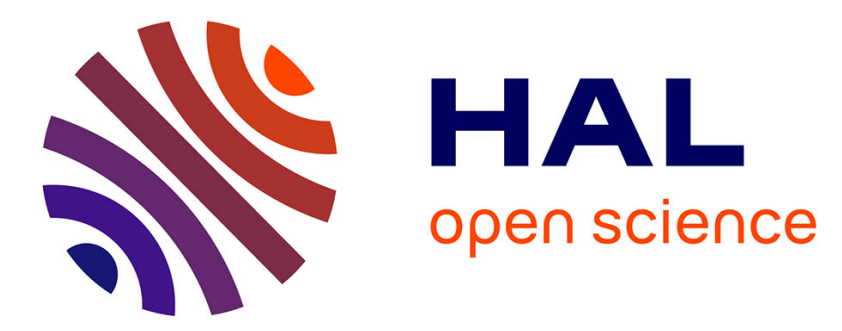

\title{
Computationally inexpensive free vortex method to obtain vortex position in the wake of a horizontal axis wind turbine
}

Issam Meghlaoui, Ivan Dobrev, Fawaz Massouh, A. Ouahab Benretem, Dalila Khalfa

\section{To cite this version:}

Issam Meghlaoui, Ivan Dobrev, Fawaz Massouh, A. Ouahab Benretem, Dalila Khalfa. Computationally inexpensive free vortex method to obtain vortex position in the wake of a horizontal axis wind turbine. International Journal of Fluid Mechanics Research, 2017, 44 (5), pp.427-443. 10.1615/InterJFluidMechRes.2017019852 . hal-02445464

\section{HAL Id: hal-02445464 \\ https://hal.science/hal-02445464}

Submitted on 20 Jan 2020

HAL is a multi-disciplinary open access archive for the deposit and dissemination of scientific research documents, whether they are published or not. The documents may come from teaching and research institutions in France or abroad, or from public or private research centers.
L'archive ouverte pluridisciplinaire HAL, est destinée au dépôt et à la diffusion de documents scientifiques de niveau recherche, publiés ou non, émanant des établissements d'enseignement et de recherche français ou étrangers, des laboratoires publics ou privés. 


\title{
COMPUTATIONALLY INEXPENSIVE FREE VORTEX METHOD TO OBTAIN VORTEX CORE POSITION IN THE WAKE OF A HORIZONTAL AXIS WIND TURBINE
}

\author{
Issam Meghlaoui, ${ }^{1,3, *}$ Ivan Dobrev, ${ }^{2}$ Fawaz Massouh, ${ }^{2}$ \\ A. Ouahab Benretem, ${ }^{3}$ \& Dalila Khalfa ${ }^{3}$
}

\author{
${ }^{1}$ Faculty of Sciences and Technology, Bachir el Ibrahimi University BBA, El-Anasser, 34030, \\ Algeria \\ ${ }^{2}$ DynFluid Laboratory, Arts et Métiers-ParisTech, 151, bd L'Hôpital, Paris, 75013, France \\ ${ }^{3}$ Faculty of Engineering Sciences, Badji Mokhtar-Annaba University, P.O. Box 12, 23000 \\ Annaba, Algeria
}

\begin{abstract}
*Address all correspondence to: Issam Meghlaoui, Faculty of sciences and Technology, Bachir el Ibrahimi University BBA, El-Anasser, 34030, Algeria, E-mail: issameghlaoui@yahoo.fr
\end{abstract}

\begin{abstract}
This work aims to develop a free wake model, allowing quick simulation of flow through a horizontal axis wind turbine. The rapidity of computation is particularly interesting when this aerodynamic model is integrated with complementary mechanical and electrical models in order to study the unsteady behavior of the complete chain of energy transfer in the wind turbine. The proposed model takes into account both the tangential and longitudinal vorticity of the vortex system formed behind the rotor. The employed vortex system replaces the helical wake close to the wind turbine by a series of vortex rings and the far wake by a semi-infinite vortex cylinder. By taking into account the root vortex, the proposed model is used to study the development of the near wake of a horizontal axis wind turbine for different speeds of rotation. The shape of the near wake as well as the position of the trailing vortices are compared with particle image velocimetry experimental results for a low-power wind turbine tested in the wind tunnel of Arts et Métiers-ParisTech. The model shows a good agreement between the calculation and the experience.
\end{abstract}

KEY WORDS: free wake, induced velocity, PIV, root vortex, semi-infinite vortex cylinder, tip vortex, vortex ring, wind turbine wake

\section{INTRODUCTION}

Although nowadays there is widespread use of CFD simulations, models based on the vortex theory are still in use, especially in the studies of helicopter rotors operating in hover and vertical flight, as well as for the wake modeling of wind turbines. As shown in the literature, for an axisymmetric flow, as in the case of flow around the wind turbine rotor, the vortex models have an advantage relative to CFD simulations with regard to the speed of the calculation with a comparable degree of precision.

Indeed, the extraction of energy from the wind by the wind turbine implies a deceleration of the flow and the creation of a helical wake that develops behind the rotor, trailing from both the tip and the root of the blade. It becomes interesting to reduce the complexity of the calculation of the flow through the wind turbine by replacing this vortex structure with an equivalent vortex system, composed of simple vortex elements, for which we know the exact solution for the induced velocities at any point in the flow field, such as the vortex ring, semi-infinite vortex lines, and cylinders.

In this context, the representation of the wake of the rotors with vortex rings was first used to model the wake of helicopters. A recent work of Karpatne et al. (2014) models the free wake of the rotor of a helicopter with a series of vortex rings in order to study the aperiodic movement of the tip vorticities observed in the wake of a helicopter in 


\begin{tabular}{|c|c|c|c|}
\hline \multicolumn{4}{|c|}{ NOMENCLATURE } \\
\hline$a_{j}$ & local axial induction factor & \multirow[t]{3}{*}{$V_{i r}$} & radial induced velocity by \\
\hline$a_{j}^{\prime}$ & local tangential induction factor & & the vortexrings on the $i$ th \\
\hline$C l(j)$ & local lift coefficient & & vortex ring $[\mathrm{m} / \mathrm{s}]$ \\
\hline$C p$ & power coefficient, efficiency & \multirow[t]{3}{*}{$V_{i z}$} & axial induced velocity by the \\
\hline$C t$ & thrust coefficient & & vortex rings on the $i$ th \\
\hline$c(j)$ & chord length of a rotor blade $[\mathrm{m}]$ & & vortex ring $[\mathrm{m} / \mathrm{s}]$ \\
\hline$E(s(z))$ & $\begin{array}{l}\text { complete elliptic integrals of } \\
\text { second kind }\end{array}$ & $V_{r}$ & $\begin{array}{l}\text { radial induced velocity by the } \\
\text { vortex ring }[\mathrm{m} / \mathrm{s}]\end{array}$ \\
\hline$K(s(z))$ & $\begin{array}{l}\text { complete elliptic integrals of } \\
\text { first kind second }\end{array}$ & $V_{T}$ & $\begin{array}{l}\text { total tangential induced velocity } \\
\text { by the root vortex }[\mathrm{m} / \mathrm{s}]\end{array}$ \\
\hline$N$ & number of rotor blades & \multirow[t]{2}{*}{$V_{t}$} & tangential induced velocity by a \\
\hline$p$ & vortex pitch distance $[\mathrm{m}]$ & & seriesof vortex segment $[\mathrm{m} / \mathrm{s}]$ \\
\hline$R$ & blade radius $[\mathrm{m}]$ & \multirow[t]{2}{*}{$V_{z}$} & axial induced velocity by the \\
\hline$R_{r}$ & vortex ring radius $[\mathrm{m}]$ & & vortex ring $[\mathrm{m} / \mathrm{s}]$ \\
\hline$R_{c}$ & $\begin{array}{l}\text { semi-infinite vortex cylinder radius } \\
{[\mathrm{m}]}\end{array}$ & $V_{r-i n d}$ & $\begin{array}{l}\text { radial induced velocity in } \\
\text { the wake }[\mathrm{m} / \mathrm{s}]\end{array}$ \\
\hline$r_{c}$ & core radius of a vortex ring $[\mathrm{m}]$ & \multirow[t]{2}{*}{$V_{z-i n d}$} & axial induced velocity in \\
\hline \multirow[t]{2}{*}{$r_{i}^{n}$} & radial position of the $i$ th vortex ring & & the wake $[\mathrm{m} / \mathrm{s}]$ \\
\hline & at the $n$th time step [m] & $w_{j}$ & local resulting speed $[\mathrm{m} / \mathrm{s}]$ \\
\hline \multirow[t]{2}{*}{$r_{i}^{*}$} & radial position of the $i$ th vortex ring & & cylindrical coordinates $[\mathrm{m}]$ \\
\hline & at the intermediate time step $[\mathrm{m}]$ & \multirow{2}{*}{$\begin{array}{l}x, r, z \\
z_{i}^{n}\end{array}$} & axial position of the $i$ th vortex \\
\hline$s(z)$ & parameter of elliptic integral & & ring at the $n$th time step $[\mathrm{m}]$ \\
\hline$T$ & thrust $[\mathrm{N}]$ & \multirow[t]{3}{*}{$z_{i}^{*}$} & axial position of the $i$ th vortex \\
\hline$t$ & time $[\mathrm{s}]$ & & ring at the intermediate time \\
\hline$U$ & dimensionless speed & & step $[\mathrm{m}]$ \\
\hline$U_{0}$ & wind speed $[\mathrm{m} / \mathrm{s}]$ & $\overrightarrow{z_{a}}$ & vector positions of point $a$ \\
\hline$U_{r}$ & $\begin{array}{l}\text { radial velocity of a semi-infinite } \\
\text { vortex cylinder }[\mathrm{m} / \mathrm{s}]\end{array}$ & $\overrightarrow{z_{b}}$ & vector positions of point $b$ \\
\hline \multirow[t]{2}{*}{$U_{t}$} & tangential induced velocity by the & \multicolumn{2}{|c|}{ Greek Symbols } \\
\hline & semi-infinite vortex line $[\mathrm{m} / \mathrm{s}]$ & $\alpha_{j}$ & local angle of attack [rad] \\
\hline \multirow[t]{2}{*}{$U_{z}$} & axial induced velocity semi-infinite & $\gamma$ & vorticity $[\mathrm{m} / \mathrm{s}]$ \\
\hline & vortex cylinder $[\mathrm{m} / \mathrm{s}]$ & $\Gamma$ & circulation $\left[\mathrm{m}^{2} / \mathrm{s}\right]$ \\
\hline \multirow[t]{3}{*}{$U_{r i}$} & radial induced velocity by the & $\Delta t$ & blade passage time (time step) [s] \\
\hline & semi-infinite vortex cylinder on & $\theta_{j}$ & local pitch distance angle [rad] \\
\hline & the $i$ th vortex ring $[\mathrm{m} / \mathrm{s}]$ & $\Pi(s(0), s(z))$ & complete elliptic integral of \\
\hline \multirow[t]{3}{*}{$U_{z i}$} & axial induced velocity by the & & the third kind \\
\hline & semi-infinite vortex cylinder & $\rho$ & constant density $\left[\mathrm{kg} / \mathrm{m}^{3}\right]$ \\
\hline & on the $i$ th vortex ring $[\mathrm{m} / \mathrm{s}]$ & $\varphi_{j}$ & flow angle [rad] \\
\hline$V_{a}$ & axial velocity of the vortex rings $[\mathrm{m} / \mathrm{s}]$ & $\Omega$ & rotor rotational speed $[\mathrm{rad} / \mathrm{s}]$ \\
\hline
\end{tabular}

hover. Reddy and Stewart (2009), present a prescribed wake of the rotor of a helicopter by semi-infinite vortex lines that model the near wake and vortex rings to represent the intermediate and far wake. 
Modeling of the wake with a series of vortex rings was adopted for wind turbines. De Vaal et al. (2014a) show that a model based on a vortex ring is able to cope with the fluctuations in the speed and direction of the wind for the case of a floating wind turbine. Baldacchino and Van Bussel (2014) present a model based on the vortex ring that allows a useful overview of the effects of the wake in response to external disturbances such as shear, yawing, and ground effect.

The near wake plays an important role in the assessment of the aerodynamic performance of the wind rotor. Meanwhile, it allows one to define the initial conditions of development of the far wake.

The vorticity in the far wake can be modeled in different manners. Reddy and Stewart (2009) model the helicopter rotor far wake with a single vortex ring that has the same diameter as the rotor and which moves simultaneously with the last vortex ring of the intermediate wake. Van Heemst et al. (2015) coupled the vortex rings model for the near wake of a wind turbine with the more elaborate deficit model for the far wake of Jensen and Larsen.

In this paper the near wake is represented by a series of vortex rings free to move and to interact with each other, while the far wake is represented by a semi-infinite vortex cylinder. This coupling leads to a model with good accuracy with regard to the total velocity field of the wake. As for the root vortex of the blade, it was modeled in the work of De Vaal et al. (2014b) by a series of vortex ring. In the work of Branlard and Gaunaa (2015), the root vortex of the blade is modeled by a semi-infinite vortex line. To complete the representation of our model, the longitudinal vorticity, represented by the root vortex of the blade, will be modeled by a series of vortex segments for the near wake and a semi-infinite vortex line that models the far wake.

The authors of this article are particularly interested in the development of a reliable numerical model, based on the vortex theory, which will be able to obtain the shape of the wake behind the rotor, to determine the position of blade tip vortices, and to calculate the axial and radial induced velocities at any point of the wake. This model will be validated using experimental data obtained in the wind tunnel at Arts et Métiers-ParisTech where flow around a three-bladed horizontal axis wind turbine is investigated by particle image velocimetry (PIV).

\section{PRESENTATION OF THE MODEL}

An idealized wind turbine wake model is introduced where the induced velocities in the rotor plane are determined by modeling the influence of the helical wake that develops behind the rotor, by considering the rotor blades as lifting lines, and also free vortices with a constant intensity $\Gamma$, which is trailed both at the root and ant the tip of the blade, such as shown in Fig. 1

The proposed model replaces the near wake of the wind turbine with a series of vortex rings and the far wake with a semi-infinite vortex cylinder, while the root vortices segments of the blades combine to form a single vortex along the axis of the rotor, which is replaced by a vortex line of intensity $N \Gamma$, as shown in Fig. 2. The latter is primarily responsible for the induction of the tangential velocity.

\section{INDUCED VELOCITIES FROM THE DIFFERENT COMPONENTS OF THE VORTEX SYSTEM}

In this section are presented in detail all the components of the vortex system and their induced velocities. The influence of each component is discussed in Section 4.

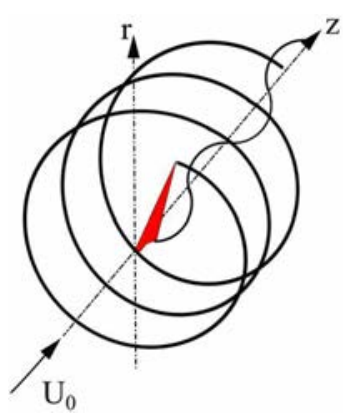

FIG. 1: Representation of the helical wake of a wind turbine 


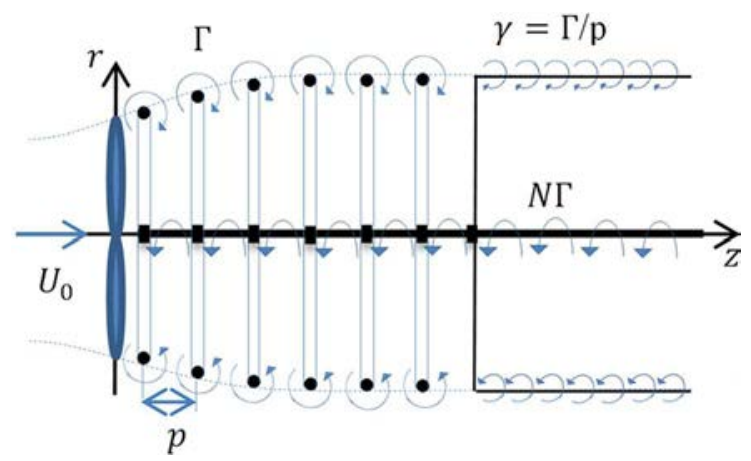

FIG. 2: Vortex system replacing the helical wake of the wind turbine

\subsection{Induced Velocities from the Series of Vortex Rings}

The near helical wake of the rotor is approximated with a series of vortex rings, free to move axially and to expand or contract radially, interacting under the effect of the induced velocity field (see Fig. 3). Each time step $\Delta t$ corresponds to the detachment of a vortex ring of $\Gamma$ intensity that is proportional to the rotor thrust $T$. The pitch distance $p$, which separates two rings, is calculated from the axial induced velocity in the rotor plane. For each time step a predictorcorrector scheme of second order is used to update both the radial and axial position of the vortex rings, calculated by the induced velocity field in the wake.

This series of vortex rings induces at the plane of the rotor an axial resulting velocity opposite to the upstream wind direction. The velocity field determines the radial and axial position of the vortex rings in the wake of the wind turbine.

The vortex rings are placed at a distance $d$ behind the rotor. This distance is determined by a comparison of the induced velocities in the rotor plane between a helical wake and an equivalent series of vortex rings. The vortex rings are allowed to move freely and to burst under the effect of the induced velocity field in the wake. The cross section of the rings is considered circular at any moment; thus the axial and radial velocities induced by the series of vortex rings is calculated according to the Biot-Savart law (Karpatne et al., 2014):

$$
V_{z}(r, z)=-\frac{\Gamma}{4 \pi} \int_{0}^{2 \pi} \frac{R_{r}\left(R_{r}-r \cos (\theta)\right)}{\left(R_{r}^{2}+r^{2}-2 r R_{r} \cos (\theta)+z^{2}\right)^{3 / 2}} d(\theta)
$$

This exact solution is also expressed by the elliptic integrals of first and second type at any point of the flow field (Van Heemst et al., 2015). Thus, for a vortex ring with radius $R_{r}$ and intensity $\Gamma$, the axial $V_{z}$ and radial $V_{r}$ components of

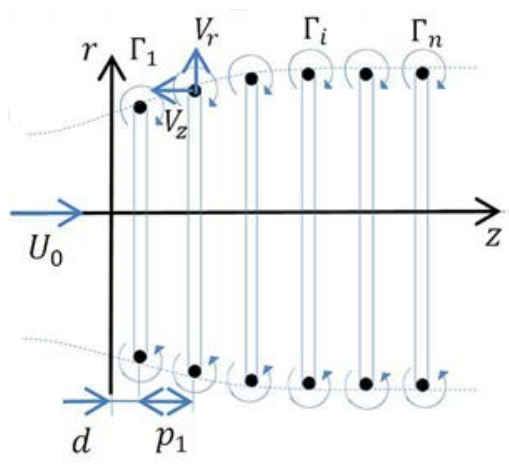

FIG. 3: Series of vortex rings modeling the near wake 
the induced velocities are expressed by Eqs. (2) and (3), respectively:

$$
\begin{aligned}
& V_{z}(r, z)=-\frac{\Gamma}{2 \pi \sqrt{z^{2}+\left(r+R_{r}\right)^{2}}}\left[K(s(z))+\frac{R_{r}^{2}-r^{2}-z^{2}}{z^{2}+\left(r-R_{r}\right)^{2}} E(s(z))\right] \\
& V_{r}(r, z)=-\frac{z \Gamma}{2 \pi \sqrt{z^{2}+\left(r+R_{r}\right)^{2}}}\left[K(s(z))-\frac{R_{r}^{2}+r^{2}+z^{2}}{z^{2}+\left(r-R_{r}\right)^{2}} E(s(z))\right]
\end{aligned}
$$

where $K(s)$ and $E(s)$ are elliptic integrals of the first and second kind, respectively, expressed in terms of parameter $s(z)$ :

$$
s(z)=\frac{4 r R_{r}}{z^{2}+\left(r+R_{r}\right)^{2}}
$$

It is considered that induction occurs at point $\mathbf{M}$ with coordinates $\mathbf{M}\left(r_{m}, z_{m}\right)$ by a vortex element located at the point $\mathrm{N}$ with coordinates $\mathrm{N}\left(r_{n}, z_{n}\right)$. Lewis (2005) introduced the nondimensional coordinates $r \mathrm{r}$ and $z \mathrm{z}$ to the point where we want to calculate the induced velocity, calculated by Eqs. (5) and (6):

$$
\begin{gathered}
r=\frac{r_{m}}{r_{n}} \\
z=\frac{z_{m}-z_{n}}{r_{n}}
\end{gathered}
$$

It is noted that $r$ and $z$ are dimensionless only for the calculation of the induced velocities by the vortex rings and the semi-infinite vortex cylinder. For all other applications, when used with indexes $r_{i}$ and $z_{i}$, they are measured in [m] and respectively represent the radial and axial coordinate.

The velocity field induced by a series of four vortex rings with the same diameter and vortex intensity $\Gamma=-1$ $\left[\mathrm{m}^{2} / \mathrm{s}\right]$, placed at a distance fixed relative to each other, is represented in Fig. 4. The streamlines created by this series of vortices are almost parallel to the axis $z / R$ in the region $-1<r / R<1$ and $-4<z / R<0$, similarly to the vortex tube. It must be noted that induced velocity becomes very important near the vortex core, where high velocity gradients are observed. For this reason the displacement of the vortex core at each time step is calculated by an algorithm based on the predictor-corrector method, which permits one to avoid the collision between vortices.

\subsection{Induced Velocities by the Semi-Infinite Vortex Cylinder}

To complete the representation of the proposed model, a far wake modeling is needed in order to make the model more realistic in terms of the form of the wake behind the rotor. As shown Fig. 5, it is proposed to couple the vortex system that models the near wake with a semi-infinite vortex cylinder, which replaces the far wake.

Both the axial and radial components of the induced velocity by the semi-infinite vortex cylinder with radius $R_{c}$ and vorticity $\gamma$ are discussed in the work of Lewis (2005) and Branlard and Gaunaa (2015). For this study,

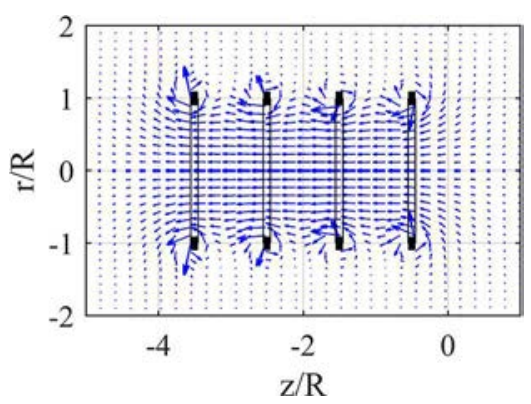

FIG. 4: Velocity field induced by four vortex rings of the same diameter and vortex intensity, $R_{r}=R$ 


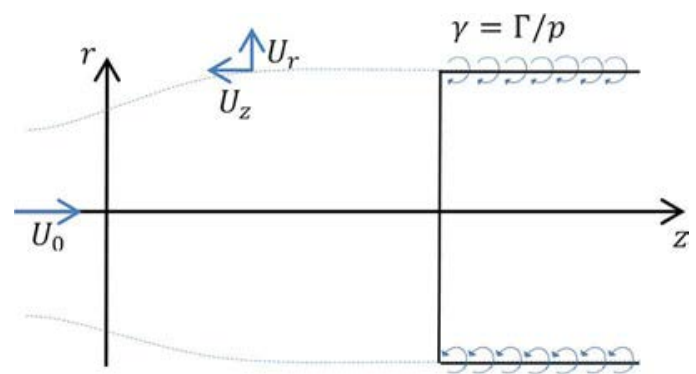

FIG. 5: Semi-infinite vortex cylinder, modeling the far wake of the wind turbine

the expression for the radial induced velocity $U r$ is Eq. (7), and for the axial $U_{z}$ it is Eqs. (8) and (9), for any nondimensional coordinate $(r, z)$ in the explored velocity field:

$$
U_{r}(r, z)=-\frac{\Gamma}{2 \pi} \sqrt{\frac{R_{c}}{r}}\left[\frac{2-s(z)}{\sqrt{s(z)}}\left(K(s(z))-\frac{2}{\sqrt{s(z)}} E(s(z))\right)\right]
$$

If $r \neq R_{c}$ :

$$
U_{z}(r, z)=\frac{\Gamma}{2}\left[\frac{R_{c}-r+\left|R_{c}-r\right|}{2\left|R_{c}-r\right|}\right]+\frac{\Gamma}{2}\left[\frac{z \sqrt{s(z)}}{2 \pi \sqrt{R_{c} r}}\left(K(s(z))+\frac{R_{c}-r}{R_{c}+r} \prod(s(0), s(z))\right)\right]
$$

If $r=R_{c}$ :

$$
U_{z}(r, z)=\frac{\Gamma}{4}+\frac{\Gamma}{2}\left[\frac{z \sqrt{s(z)}}{2 \pi \sqrt{R_{c} r}} K(s(z))\right]
$$

where $\Pi(s(0), s(z))$ is the elliptic integral of the third kind, $K(s(z)), E(s(z)), s(z), r$, and $z$ are calculated in the same way as in the case of the vortex rings.

The velocity field induced by the semi-infinite vortex cylinder with the same diameter as that of the last vortex ring that forms the near wake, with vorticity $\gamma=-1[\mathrm{~m} / \mathrm{s}]$, is presented in Fig. 6 . The semi-infinite vortex cylinder extends axially over a distance $0<z<\infty$. The streamline pattern is directly analogous to the magnetic flux induced by a semi-infinite solenoid. Note that the streamlines have some curvature at the entrance of the vortex tube, and they become progressively parallel to the tube as $z \rightarrow \infty$. Note also that the velocity induced by this vortex tube is relatively small upstream and then its effect is negligible in the rotor plane. This semi-infinite vortex cylinder is placed at a fixed distance behind the last vortex ring, very far from the wind turbine rotor, and its location is determined by the shape of the wake.

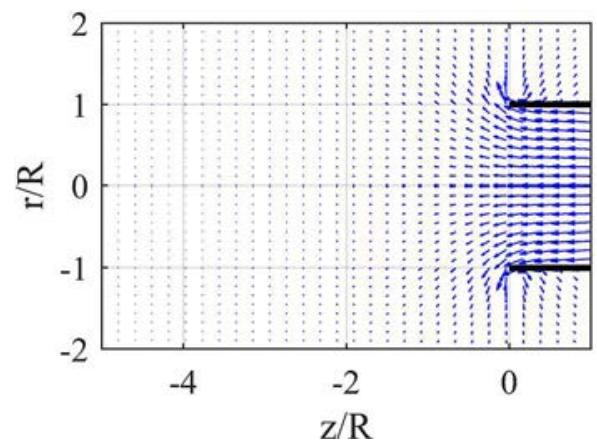

FIG. 6: Velocity field induced by the semi-infinite vortex cylinder 
Figure 7 shows the coupling between the semi-infinite vortex cylinder and the series of vortex rings, as well as the total induced velocity field by the proposed vortex system. Note that the radius is the same for the vortex rings and the semi-infinite vortex cylinder, $R_{r}=R_{c}$.

The distance between the last vortex ring and the semi-infinite vortex cylinder is determined such to insure a minimum of distortion of the streamlines. It must be noted that the diameter of the vortex tube which was calculated at the previous time step has its influence on the shape of the far wake.

\subsection{Induced Velocity by the Root Vortex of the Blade}

The root vortices of the blades are modeled in the near wake by a series of vortex segments of intensity $N \Gamma$ and by a semi-infinite vortex line in the far wake, as shown in Fig. 8. This vortex system permits one to represent the tangential velocity in the wake and in the rotor plane. The formula of Biot-Savart is used to calculate the tangential induced velocity $V_{t}$. If we consider a point on the blade, then the velocity induced by the vortex segment $a-b$, shown in Fig. 8 , can be expressed as

$$
\vec{V}_{t}=\frac{N \Gamma}{4 \pi}\left(\frac{1}{\left|\overrightarrow{z_{a}}\right|}+\frac{1}{\left|\overrightarrow{z_{b}}\right|}\right)\left[\frac{\left(\overrightarrow{z_{a}} \Lambda \overrightarrow{z_{b}}\right)}{\left|\overrightarrow{z_{a}}\right| \cdot\left|\overrightarrow{z_{b}}\right|+\overrightarrow{z_{a}} \cdot \overrightarrow{z_{b}}}\right]
$$

where $\overrightarrow{z_{a}}$ and $\overrightarrow{z_{b}}$ are the vectors, defined between the considered point and the extremities $a$ and $b$ of the vortex segment.

In the far wake, the rest of the root vortex of the blade is replaced by a semi-infinite vortex line, such as shown in Fig. 8. The expression for the tangential induced velocity $U_{t}$ of the semi-infinite vortex line, discussed in the work of Branlard and Gaunaa (2015), is given by Eq. (11). The induced velocity is purely tangential.

$$
U_{t}(r, z)=-\frac{N \Gamma}{4 \pi r}\left[1+\frac{z}{\sqrt{r^{2}+z^{2}}}\right]
$$

The total tangential induced velocity $V_{T}$ is the sum of velocity induced by the series of vortex segments $V_{t}$ and the velocity induced by the semi-infinite vortex filament $U_{t}$. This velocity is always negative and opposite to the speed of rotation of the rotor. Note that the tangential induced velocity is very important at the blade root, while at the blade tip it is low as shown in Fig. 9.

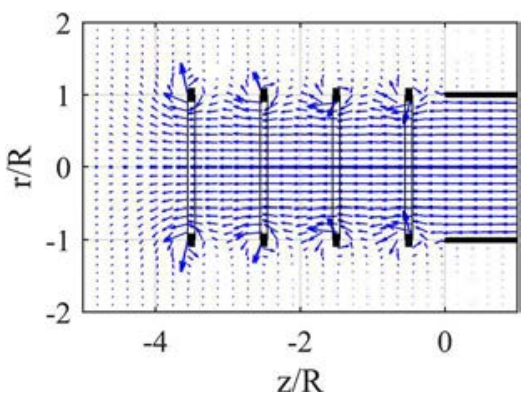

FIG. 7: Induced velocity field by the series of vortex rings and the semi-infinite vortex cylinder

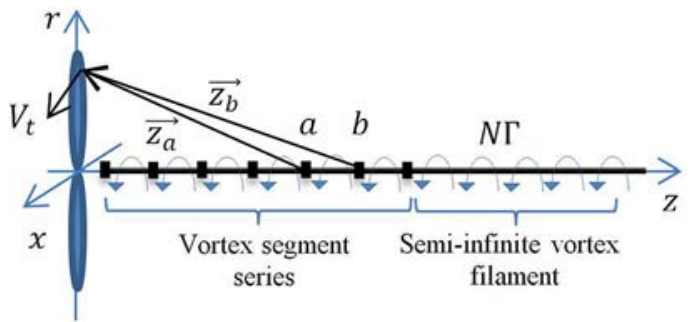

FIG. 8: Blade root vortex system 


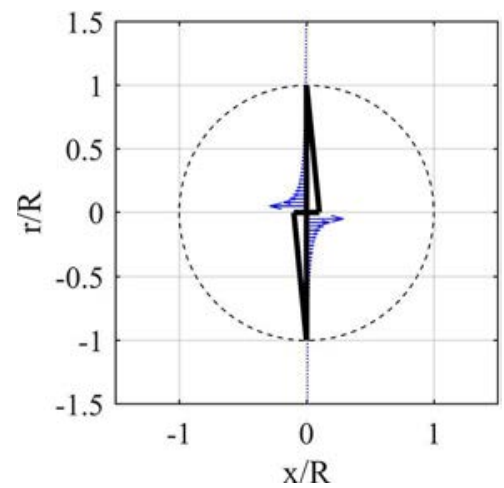

FIG. 9: Profile of the total tangential velocity induced by the root vortex

\subsection{The Blade Element Model}

The rotor blade is discretized into $K$ number of major segments. The axial and tangential induced velocities by the vortex system are evaluated on each segment, demonstrated by Karpatne et al. (2014). This affects the local blade angle of attack $\alpha_{j}$, as shown in the triangle of velocities of the blade element of index $j$, represented in Fig. 10. The flow angle is determined from the triangle of velocities and it is expressed by Eq. (12):

$$
\phi_{j}=\tan ^{-1}\left(\frac{U_{0}\left(1-a_{j}\right)}{\Omega r_{j}\left(1+a_{j}^{\prime}\right)}\right)
$$

Finally the local angle of attack is given by Eq. (13):

$$
\alpha_{j}=\phi_{j}-\theta_{j}
$$

where $\theta_{j}$ is the pitch angle of the blade element of index $j$.

The axial induction factor $a_{j}$ is calculated from the sum of the axial induced velocities by both the series of vortex rings and the semi-infinite vortex cylinder on the blade element of index $j$, according to Eq. (14):

$$
a_{j}=\frac{V_{z_{j}}+U_{z_{j}}}{U_{0}}
$$

Similarly, the tangential induction factor is calculated from the sum of the tangential induced velocities by the series of vortex segments, as well as by the semi-infinite vortex line, on the blade element of index $j$, according to Eq. (15):

$$
a_{j}^{\prime}=\frac{V_{T_{j}}}{\Omega R}
$$

The wake induces velocities on each cross section of the blade. The origin of those velocities is the development of the aerodynamic load on the blades because they lead to a change in the value of the flow angle $\varphi(j)$ on each cross

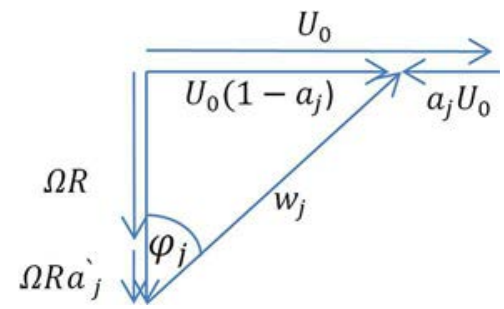

FIG. 10: Triangle of velocities for an element of the blade 
section of the blade. The local lift coefficient $C l(j)$ is calculated for each $\alpha_{j}$ after a consultation with the data table of the blade aerodynamic profile. The total thrust $T$ produced by the rotor is given by

$$
T=\frac{1}{2 K} \sum_{j}^{K} N \rho w_{j}^{2} C l(j) c(j) \Delta r
$$

where $j$ is the number of the element of the blade, and $w_{j}$ is local resulting speed. The slightest variation in time of the axial force (the total thrust) implies a variation of the vortex intensity $\Gamma$ for the next vortex ring that will be emitted after one time step $\Delta t$. The Kutta-Joukowski theorem is used to evaluate the vortex intensity for each blade. The equivalent vortex intensity can be calculated from the total thrust generated by the wind turbine.

$$
T=\Gamma \rho \int_{0}^{R} \Omega r d r
$$

By adding the total thrust from all blades and assuming that all resulting circulation is trailed from the tip of the blades, the intensity of the emitted vortex ring is given by

$$
\Gamma=\frac{2 T}{\rho N R^{2} \Omega}
$$

where $\Gamma$ is the circulation of the vortex ring that will be emitted. This iterative calculation process for $\Gamma$ is made at each time step $\Delta t$, as demonstrated by Karpatne et al. (2014).

The distance travelled by the emitted vortex ring before the next pass of the blade is denoted with $p$. This distance is obtained taking into account the time step $\Delta t$ between blade passages. De Vaal et al. (2014b) assume that the tangential induction factor is negligible in the vicinity of the tip of the blade $a_{j}^{\prime} \approx 0$, where $j=K$ because of its distance from the center of the root vortex (Fig. 9), from where a simplification of the representation of the triangle of velocities can be made. The time step $\Delta t$ is expressed with Eq. (19):

$$
\Delta t=\frac{2 \pi}{\Omega N}
$$

Thus, the pitch distance $p$ that separates two successive rings is given by Eq. (20):

$$
p=\frac{2 \pi U_{0}\left(1-a_{k}\right)}{\Omega N}
$$

\section{UNSTEADY WAKE-CASE TRAVEL SPEED}

In the stationary case all rings have constant vortex intensity, while during an unsteady regime, the vortex intensity $\Gamma$ and the pitch distance $p$ of the emitted rings are functions of the instantaneous load of the wind turbine. Figure 11 represents the wake behind the wind turbine during a transition. The issued vortices have a vortex intensity $\Gamma$ and step $p$ different from those which corresponded to the stationary regime. In each case the positions of the vortices are determined by the total velocity field in the wake. This total velocity field is the sum of the induced velocity fields, created by the series of vortex rings and by the semi-infinite vortex cylinder, the latter of which has the same diameter and moves with the same axial velocity as the last vortex ring Fig. 7, proposed by Reddy and Stewart (2009).

In the free wake, the axial position of the vortex ring is determined by summation of the wind speed $U_{0}$, the mutually induced velocities, and the self-induced velocity on a control point, situated on the vortex ring, proposed by Van Heemst et al. (2015). In this case the contribution of the far wake adds to the expression and gives the following equation:

$$
V_{a_{-} i}=U_{0}+\sum_{i=1}^{j} V_{z i}+V_{a u t i_{i}}+U_{z i}
$$




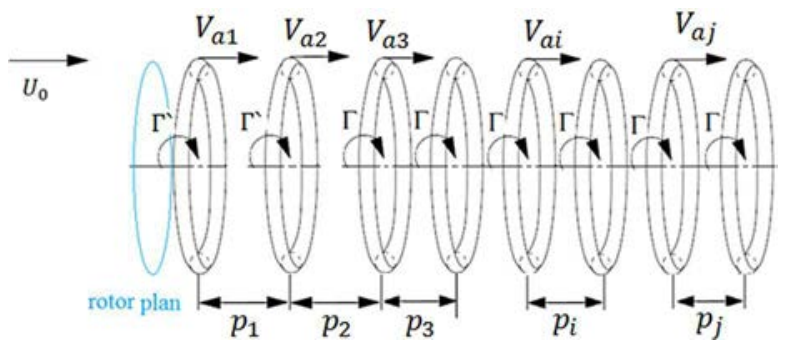

FIG. 11: Axial velocity of the vortex rings in the wake

In literature sources (Baldacchino and Van Bussel, 2014; Van Heemst et al., 2015), the self-induced velocity is calculated by applying Eq. (2) at the center of the ring. For our case, the self-induced velocity $\left(V_{a u t i}\right)$ is calculated in the control point located on the vortex ring with radius $R$ and core radius $r_{c}$, shown in Fig. 12, according to Eq. (22), proposed by Lewis (2005):

$$
V_{\text {auti }}=\frac{\Gamma}{4 \pi R_{r}}\left(\log \frac{8 R_{r}}{r_{c}}-\frac{1}{4}\right)
$$

The radial expansion or contraction velocity of the vortex ring is determined in a control point situated in the periphery of the vortex ring by summing all radial mutually induced velocities and the radial velocity induced by the semi-infinite vortex cylinder, given with Eq. (23):

$$
V_{r a_{-} i}=\sum_{i=1}^{j} V_{r i}+U_{r i}
$$

In Fig. 13, it is noticeable that the vortex rings, situated just behind the rotor, are located in an area of high radial velocities, which allows the rapid increase of their diameters while moving away from the rotor. Those radial velocities, which are determined in a control point located on the periphery of the vortex ring, are decreasing until they become zero, which means that the diameter of the rings remains constant. This, in the end, gives the actual shape of the wake of the wind turbine. This is achieved through modeling the far wake of the wind turbine with the semi-infinite vortex cylinder, which pushes the last few vortex rings to expand radially by increasing their diameters.

Similarly, Fig. 14 shows the axial velocity on the periphery of the rings based on the distance $z$ from the rotor plan. It is noticeable that the vortex rings behind the rotor initially move slower than those that are positioned further away from the plane of the rotor. The distance between the vortex rings decreases toward the far wake, away from the plane of the rotor, under the effect of the semi-infinite vortex cylinder. This pulls the rings to tighten and keeps the distance that separates the vortex rings to be constant, which gives the final and real shape of the wake of the wind turbine.

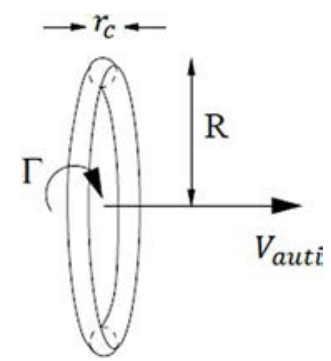

FIG. 12: Self -induced velocity by a vortex ring, $R_{r}=R$ 


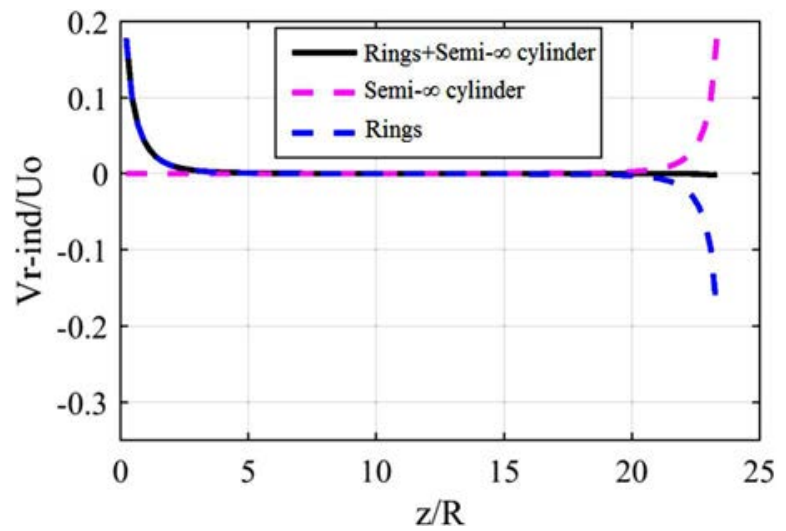

FIG. 13: The radial velocities of the vortex system in the vicinity of the vortex rings along the wake

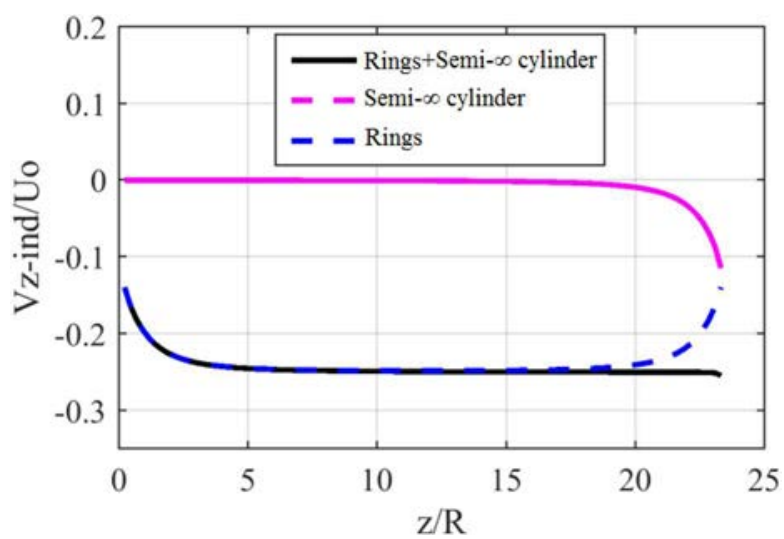

FIG. 14: The axial velocities of the vortex system in the vicinity of the vortex rings along the wake

\section{SCHEME OF TEMPORAL INTEGRATION}

In order to determine the axial position and the radial expansion or contraction of the vortex ring, the velocity field in the wake must be updated at each time step $\Delta t$ using a trapezoidal pattern predictor-corrector. For the first iteration, Eqs. (24) and (25) are used to predict an intermediate solution of radial and axial position of the vortex rings by means of a simplified Euler scheme:

$$
\begin{aligned}
& r_{i}^{*}=r_{i}^{n}+\Delta t V_{i r}\left(r_{i}^{n}, z_{i}^{n}\right) \\
& z_{i}^{*}=z_{i}^{n}+\Delta t V_{i z}\left(r_{i}^{n}, z_{i}^{n}\right)
\end{aligned}
$$

In the second time step and for reasons of accuracy and stability, the Adams-Bashforth method is used to predict the position of the vortex rings, with Eqs. (26) and (27):

$$
\begin{aligned}
& r_{i}^{*}=r_{i}^{n}+\frac{3}{2} \Delta t V_{i r}\left(r_{i}^{n}, z_{i}^{n}\right)-\frac{1}{2} \Delta t V_{i r}\left(r_{i}^{n-1}, z_{i}^{n-1}\right) \\
& z_{i}^{*}=z_{i}^{n}+\frac{3}{2} \Delta t V_{i z}\left(r_{i}^{n}, z_{i}^{n}\right)-\frac{1}{2} \Delta t V_{i z}\left(r_{i}^{n-1}, z_{i}^{n-1}\right)
\end{aligned}
$$

In this "corrector" stage, the average between the previous value $(n)$ and the predicted value is used to calculate the radial and axial position of the vortex rings using the Eqs. (28) and (29). To increase the accuracy of the results in this "corrector" stage, an iteration process is used with more than one iteration for each time step $\Delta t$ :

$$
r_{i}^{n+1}=r_{i}^{n}+\frac{\Delta t}{2}\left[V_{i r}\left(r_{i}^{*}, z_{i}^{*}\right)+V_{i r}\left(r_{i}^{n}, z_{i}^{n}\right)\right]
$$




$$
z_{i}^{n+1}=z_{i}^{n}+\frac{\Delta t}{2}\left[V_{i z}\left(r_{i}^{*}, z_{i}^{*}\right)+V_{i z}\left(r_{i}^{n}, z_{i}^{n}\right)\right]
$$

\section{VALIDATION OF THE DEVELOPED MODEL WITH WIND TUNNEL EXPERIMENTAL DATA}

In order to validate the code for aerodynamic calculation in terms of form of the wake and the positions of the trailing vortices, PIV measurements were made in the wake of the tested wind turbine in the wind tunnel of the DynFluid Laboratory in the Arts et Métiers-ParisTech University for controlled conditions of the flow.

\subsection{The Tested Wind Turbine}

The three-bladed rotor of the horizontal axis wind turbine is a model specifically designed for testing in the wind tunnel of Arts et Métiers-ParisTech (Fig. 15). The blade of this wind turbine is designed with NACA profiles (NACA 4421 at the root and NACA 4418 for the rest of the blade). The rotor diameter is $540 \mathrm{~mm}$ and the hub diameter is $42 \mathrm{~mm}$. The pitch angle is $14^{\circ}$ at the root and $2^{\circ}$ at the blade tip. The chord at the root of the blades is $39 \mathrm{~mm}$ and $29 \mathrm{~mm}$ at the tip.

During the tests, the speed of rotation varied between 1400 and $2800 \mathrm{rpm}$ for a wind speed of $9.6 \mathrm{~m} / \mathrm{s}$. The speed of rotation is controlled by a rheostat switch connected to the output of the electric generator of the wind turbine.

The axis of rotation of the turbine is placed in the middle of the test section using a $3.7 \mathrm{~cm}$ diameter mast in order to avoid the influence of the ceiling and floor on the behavior of the wake and to allow fixed lasers on the transparent ceiling of the test section to illuminate the plane of exploration with sufficient intensity.

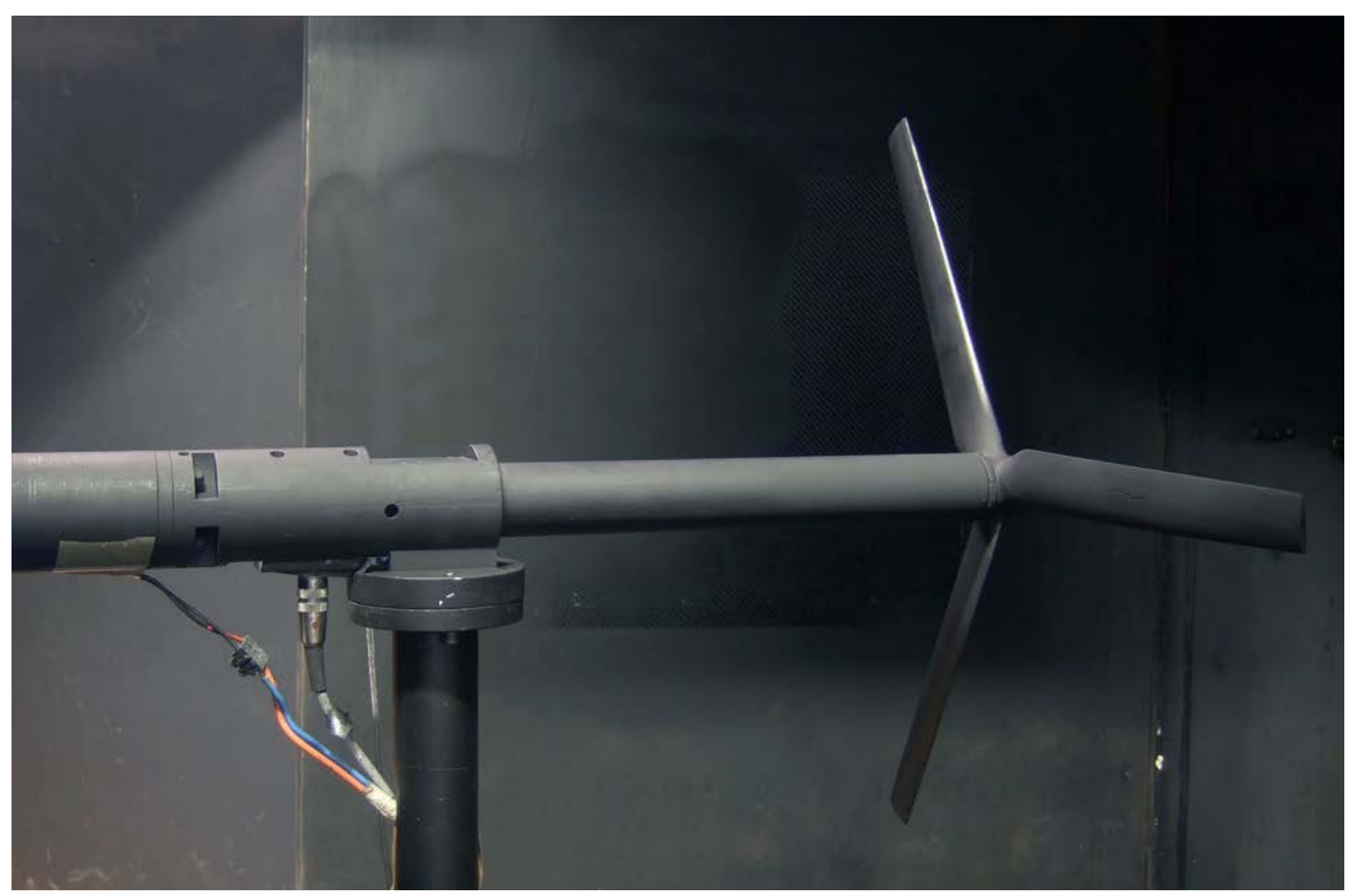

FIG. 15: Tested wind turbine in the wind tunnel 


\subsection{Measures PIV}

The shape of the vortex trailing from the tip of the blades is helical. The plane, illuminated by the laser that goes through the axis of rotation, cut these vortices. The flow within these vortices rotates with a significant angular speed. Due to the centrifugal forces, the vortex core gradually empties all its seeding particles away, and thus the core of the vortices appears on the illuminated plane as small black holes shown in Fig. 16. Note that the observed holes are emitted by the blades successively. It may be noticed that the diameter of the wake grows downstream of the rotor, which shows the deceleration of the flow due to the extraction of kinetic energy.

The acquisition of the images is managed by DynamicStudio 2.30 Dantec software, which allows the treatment of images and the computation of the average and instantaneous velocity field. Finally, this software exports results in digital form to establish an experimental database for the validation of the numerical model.

\subsection{Comparison of the Numerical Results with the Wind Tunnel Experiment}

In order to show the potential of the proposed method for the three-dimensional case, the resolution of a more difficult test case is presented, namely, the simulation of the flow around a three-bladed horizontal axis wind turbine. A comparison is made between the simulation of the wind turbine using the proposed model and the results obtained with the PIV exploration in wind tunnel.

In this experimental work, the PIV technique was used to analyze the flow downstream of a wind rotor for different speeds of rotation. At each speed, 200 pairs of images are taken at the same azimuthal position of the rotor. The treatment of images allows us to calculate the instantaneous velocity field and therefore to obtain the average velocity field for each series of images. The velocity fields help to obtain the positions of the vortices trailing from the tip of the blades and to determine the evolution of the near wake.

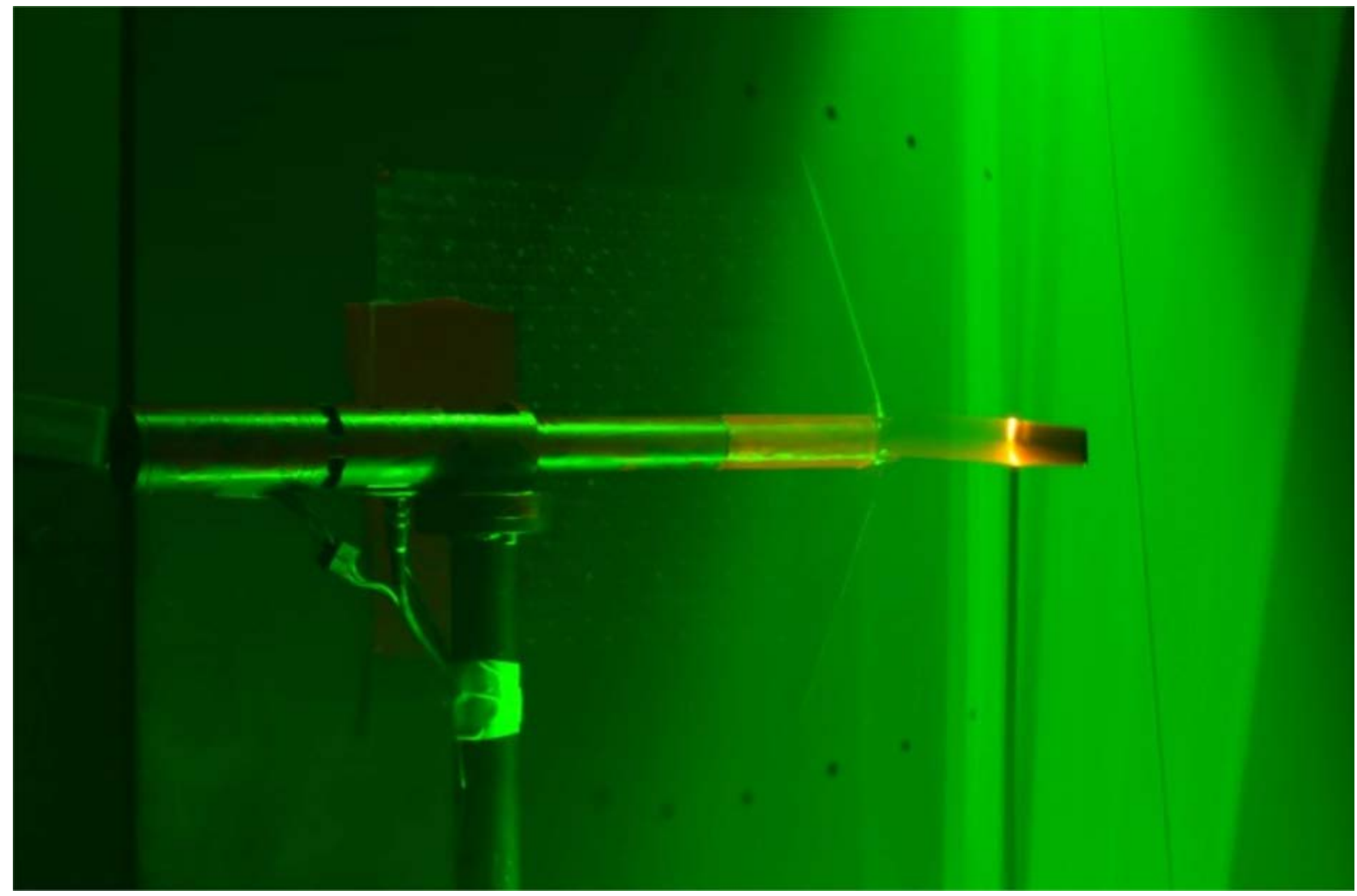

FIG. 16: PIV measurement in the wind tunnel 
On the left side of Fig. 17 are presented the numerically calculated results, while on the right side are presented the measurement which corresponds to the same regime. Figure 17 shows the dimensionless velocity field in the wake of the wind turbine for different speeds of rotation. Note that the dimensionless velocity is obtained by dividing the wake velocity by the wind tunnel speed. The velocity fields represent at left the results of computations and at right the results obtained by PIV measurements.

The averaged velocity field shows how the flow is slowed down due to the extraction of kinetic energy of the wind, according to the theory of Rankine-Froude. The results show an increasing wake radius when the speed of rotation is increased.

The iso-velocity contours represented on these fields indicate the high gradients created by the vortex cores. The positions of the vortices are obtained with satisfactory precision for the majority of cases. For some speeds of rotation and more precisely, from 1700 to $2000 \mathrm{rpm}$, the discrepancies of location become relatively important.
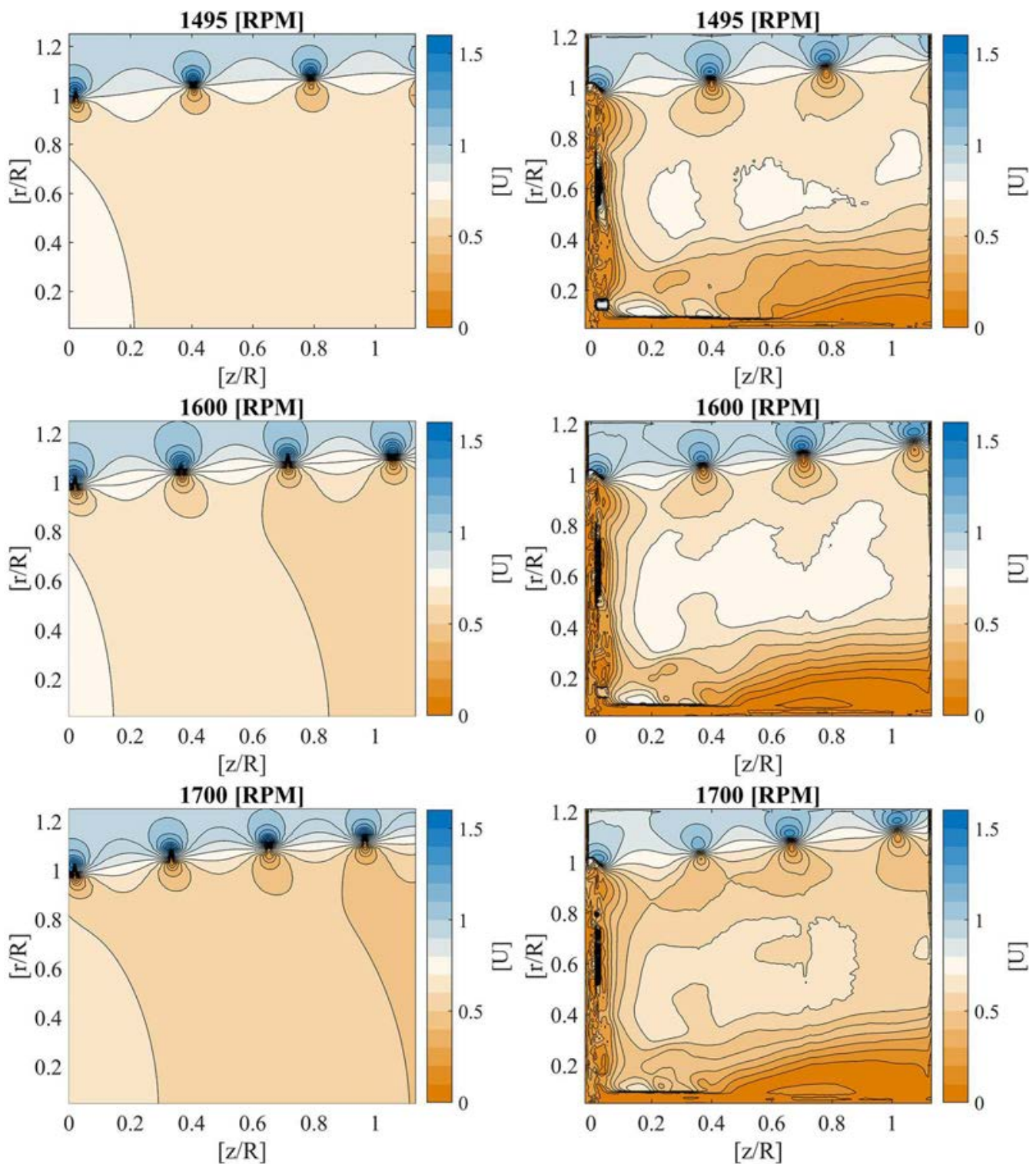

FIG. 17 

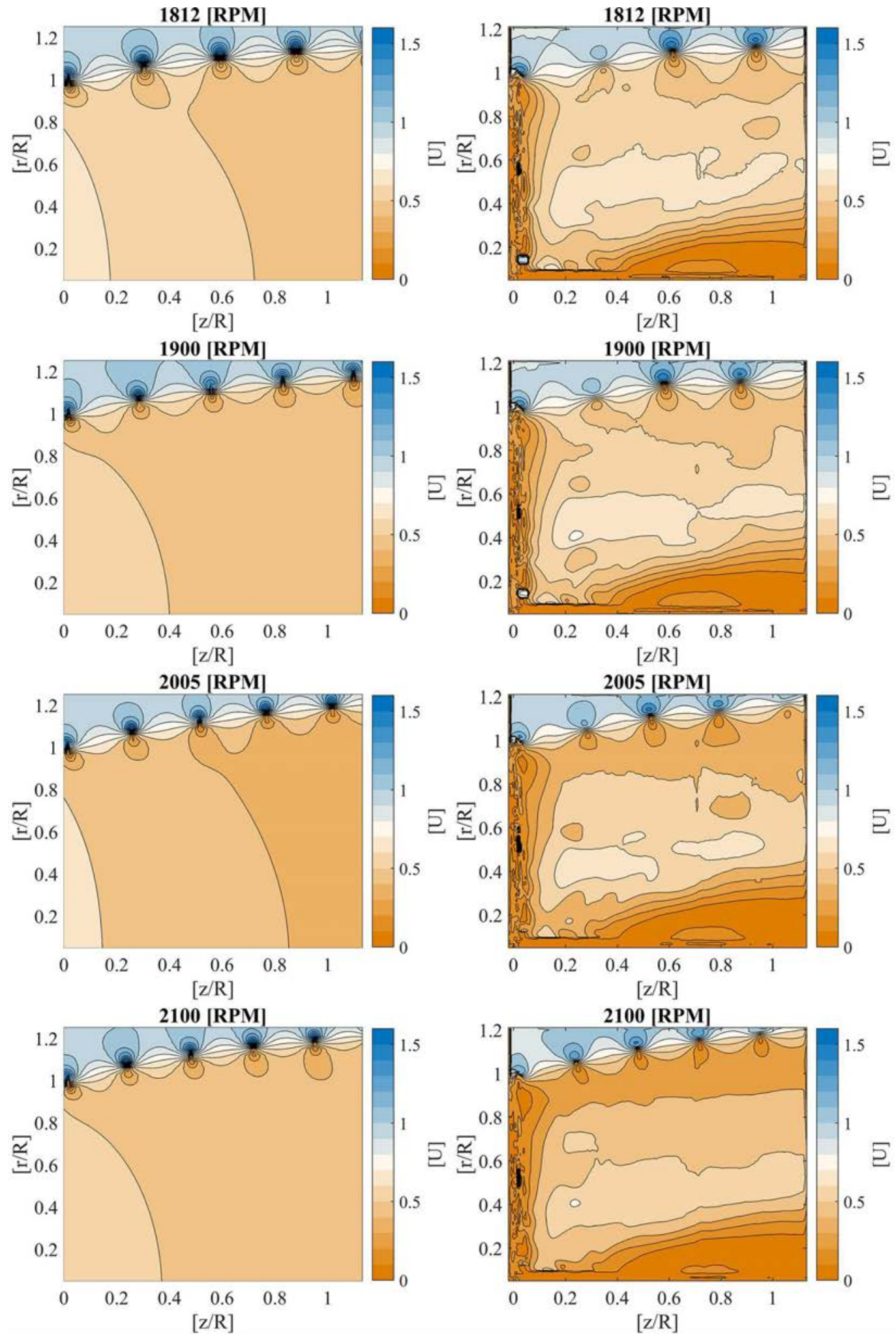

FIG. 17 

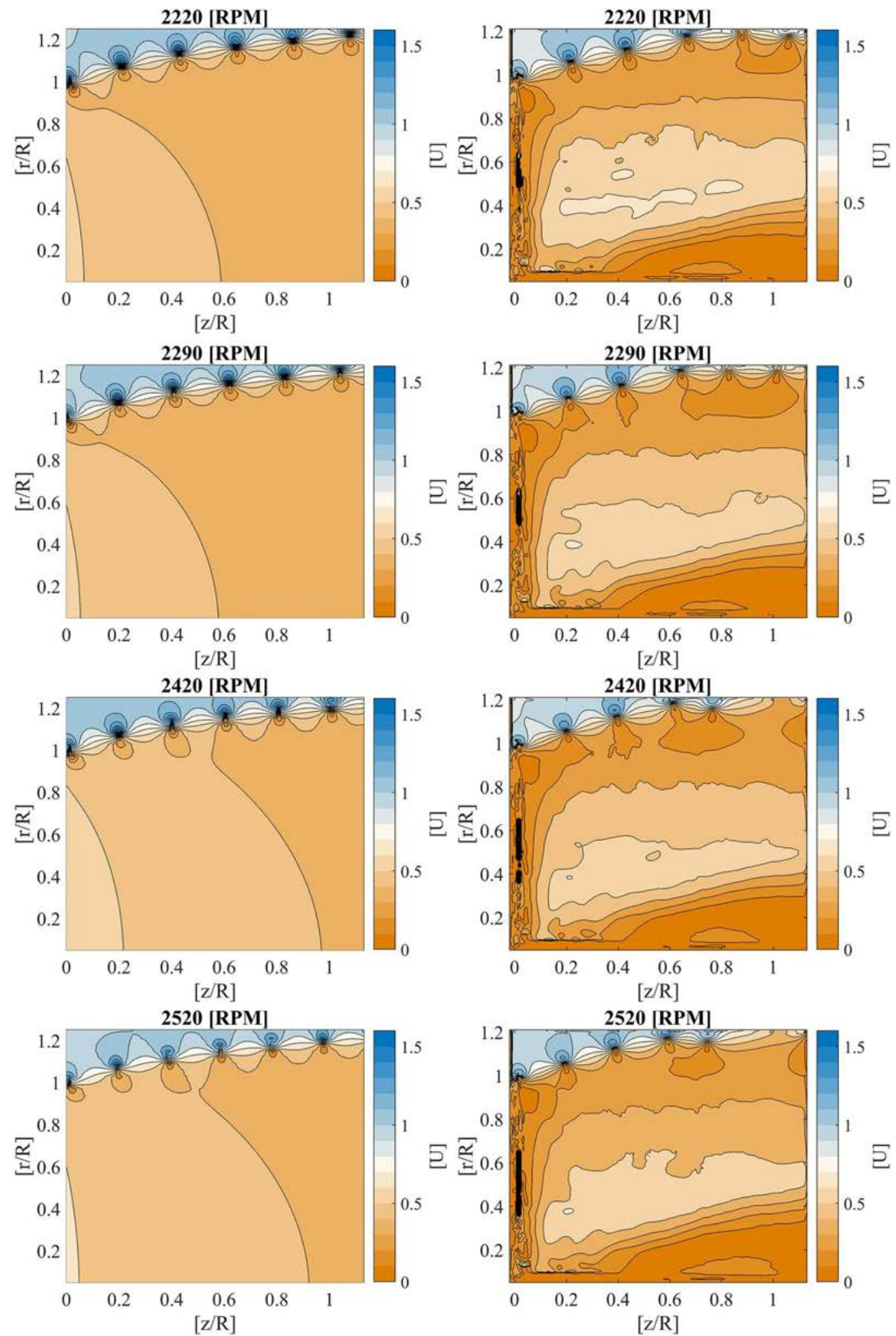

FIG. 17: Field of dimensionless velocity $U$ in the wake for different speeds of rotation: computation (left) and experimental (right). 
For speeds of rotation between 2000 and $2400 \mathrm{rpm}$, the calculated position of the trailing vortices fits well with the experimental results. Note that for speeds of rotation higher than $2400 \mathrm{rpm}$, the vortex breakdown appears at approximately $0.7 R$.

\section{CONCLUSIONS}

In this paper, a model for a horizontal axis wind turbine is proposed. The goal was to validate the use of this model to represent the flow around the wind turbine.

The developed model couples the blade element method for the calculation of aerodynamic forces and vortex model for the calculation of the induced velocity field in the plane of the rotor and downstream. The helical wake of the rotor is represented by a series of vortex rings that are allowed to freely interact with each other. The far wake is modeled by a semi-infinite vortex cylinder that moves at the same velocity as the last vortex ring constituting the near wake. The application of a predictor-corrector scheme provides accuracy and stability of the calculation. The advantage of the proposed model is to take into account the modeling of the far wake, as well as the tangential velocity, induced by the root vortex of the blade.

The simulation of the tested wind turbine by the proposed model shows that the positions of blade tip vortices are comparable to those obtained in a wind tunnel using PIV technique. Thus it is possible to define the wake border for most of the rotational speeds till the vortex breakdown. The calculation speed of the model allows real-time simulation of wind turbine behavior. This will be particularly useful if coupled with mechanical and electrical models when studying wind turbine control.

Finally, the proposed model can be considered as operational and can be used to simulate the case of an unsteadystate operation of a wind turbine rotor, which will be the subject of the next article.

\section{ACKNOWLEDGMENTS}

Issam Meghlaoui expresses most sincere gratitude to Professor Fawaz Massouh for his dedicated time and support in the process of the development of my doctoral thesis. Issam Meghlaoui is very grateful to Dr. Ivan Dobrev for his personal time and all the advice, which helped to resolve a lot of emerging problems.

\section{REFERENCES}

Baldacchino, D. and Van Bussel, G.J., Wind Turbine Wake Stability Investigations Using a Vortex Ring Modelling Approach, J. Physics Conf. Ser., vol. 555, no. 1, 012111, 2014.

Branlard, E. and Gaunaa, M., Cylindrical Vortex Wake Model: Right Cylinder, Wind Energy, vol. 18, no. 11, pp. 1973-1987, 2015.

De Vaal, J.B., Hansen, M.O., and Moan, T., Influence of Rigid Body Motions on Rotor Induced Velocities and Aerodynamic Loads of a Floating Horizontal Axis Wind Turbine, ASME 33rd Int. Conf. on Ocean, Offshore, and Arctic Eng., Ocean Renewable Energy, San Francisco, CA, vol. 9B, June 8-13, 2014a.

De Vaal, J.B., Hansen, M.O.L., and Moan, T., Validation of a Vortex Ring Wake Model Suited for Aeroelastic Simulations of Floating Wind Turbines, J. Phys. Conf. Ser., vol. 555, no. 1, 012025, 2014 b.

Karpatne, A., Sirohi, J., Mula, S., and Tinney, C., Vortex Ring Model of Tip Vortex Aperiodicity in a Hovering Helicopter Rotor, J. Fluids Eng., vol. 136, no. 7, 071104, pp. 1-9, 2014.

Lewis, R.I., Vortex Element Methods for Fluid Dynamic Analysis of Engineering Systems, Cambridge, UK: Cambridge University Press, 2005.

Reddy, K.R., and Stewart, C.J., Simulation, a Valuable Tool in the Estimation of Helicopter Flight Dynamic Characteristics, Proc. of the 18th World IMACS/MODSIM Congress, Cairns, Australia, July 13-17, 2009.

Van Heemst, J.W., Baldacchino, D., Mehta, D., and Van Bussel, G.J.W., Coupling of a Free Wake Vortex Ring Near-Wake Model with the Jensen and Larsen Far-Wake Deficit Models, J. Phys. Conf. Ser., vol. 625, no. 1, 012041, 2015. 